\title{
Incisional Hernia Post Open Appendectomy: A Case Report
}

\author{
Onyeyirichi Otuu and Ugochukwu Uzodimma Nnadozie
}

\section{ABSTRACT}

\begin{abstract}
Incisional hernia is an unusual postoperative complication after appendectomy through a McBurney's incision with an incidence of less than one percent. We present a case of incisional hernia post open appendectomy in a 40-year old woman. She presented to us with a painless right lower abdominal swelling that developed after an emergency open appendectomy for perforated appendix 7 years earlier. She was clinically stable with BMI of $24.5 \mathrm{~kg} / \mathrm{m}^{2}$. She had a non-tender, reducible mass of $20 \times 16 \times 8 \mathrm{~cm}$ protruding through a healed right iliac fossa transverse scar. She had an open onlay mesh repair with satisfactory outcome. Post appendectomy incisional hernia is uncommon. Hernioplasty offers a satisfactory outcome.
\end{abstract}

Keywords: Appendectomy, Hernioplasty, Incisional hernia, Mesh repair.

\author{
Submitted : February 19,2021 \\ Published : March 12,2021
}

ISSN: 2593-8339

DOI: $10.24018 /$ ejmed.2021.3.2.728

Dr Otuu Onyeyirichi*

MBBS, FWACS

Consultant General Surgeon,

Department of Surgery, Alex Ekwueme

Federal University Teaching Hospital

Abakaliki, Ebonyi State, Nigeria.

(e-mail: onyeyirichiotuu ${ }^{@}$ yahoo.com)

Dr Nnadozie Ugochukwu Uzodimma MBBS, FWACS, FICS

Plastic and Reconstructive Surgeon, Cosmetic plastic surgery Specialist, Hand and Micro surgeon, Division of Plastic Surgery, Department of Surgery,

Alex Ekwueme Federal University

Teaching Hospital Abakaliki, Ebonyi

State, Nigeria.

*Corresponding Author

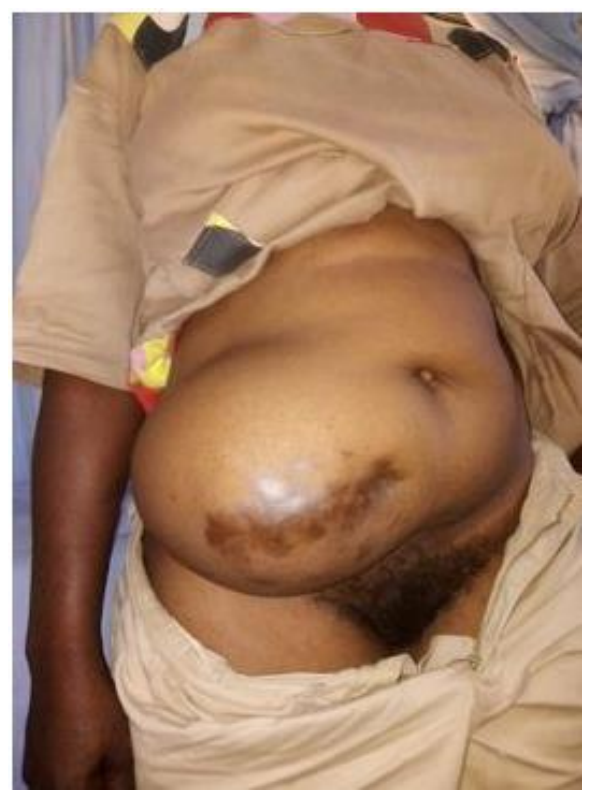

Fig. 1. The incisional hernia post open appendectomy.

The rest of the systemic examination was essentially normal. A diagnosis of post appendectomy incisional hernia was made. Patient was counseled for elective mesh repair. Her hematological parameters were within normal range likewise the chest $\mathrm{x}$-ray and serum electrolytes. Abdominal ultrasound showed a $4 \times 3 \mathrm{~cm}$ defect in the fascia with herniation of healthy bowel loops. 
Under spinal anesthesia, with prophylactic antibiotics given at induction, a transverse incision was made $2 \mathrm{~cm}$ below the scar. Findings included a large subcutaneous sac containing healthy small bowels with no appendix, attenuation of the external oblique aponeurosis and the underlying internal oblique and transversus abdominis muscles leaving a fascial defect of $4 \times 3 \mathrm{~cm}$ (Fig. 2).

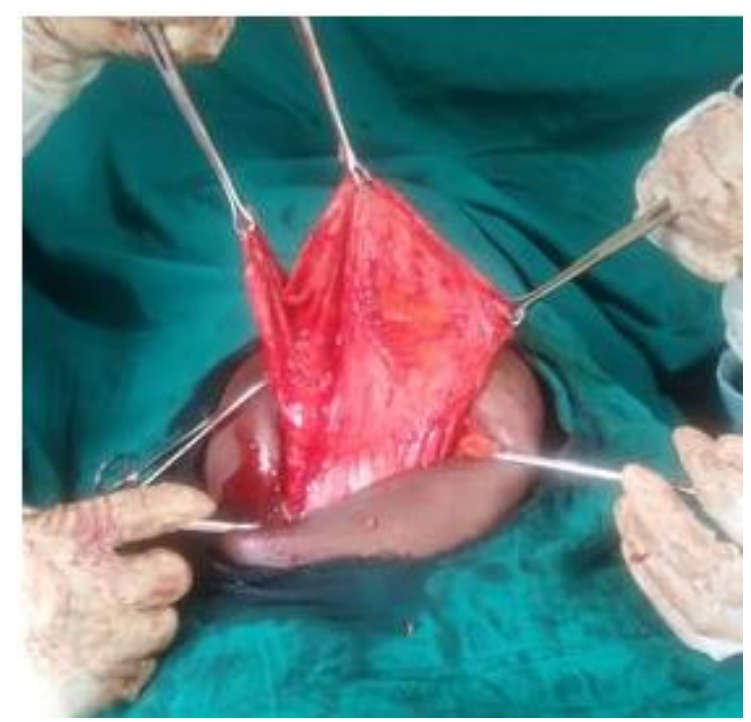

Fig. 2. The large subcutaneous sac of the incisional hernia.

The sac was excised, and the fascial edges approximated using nylon 2 sutures in a continuous fashion. A $15 \times 15 \mathrm{~cm}$ polypropylene mesh was anchored on the external oblique aponeurosis with nylon $2 / 0$ as an onlay mesh to cover the defect. Patient had an uneventful post-operative recovery and was discharged home on the $5^{\text {th }}$ post-operative day. The sutures were removed in clinic on the $10^{\text {th }}$ day. She was seen in outpatient clinic at 1 month, 6 months and 1year with no sign of recurrence and patient is satisfied with the result (Fig. 3).

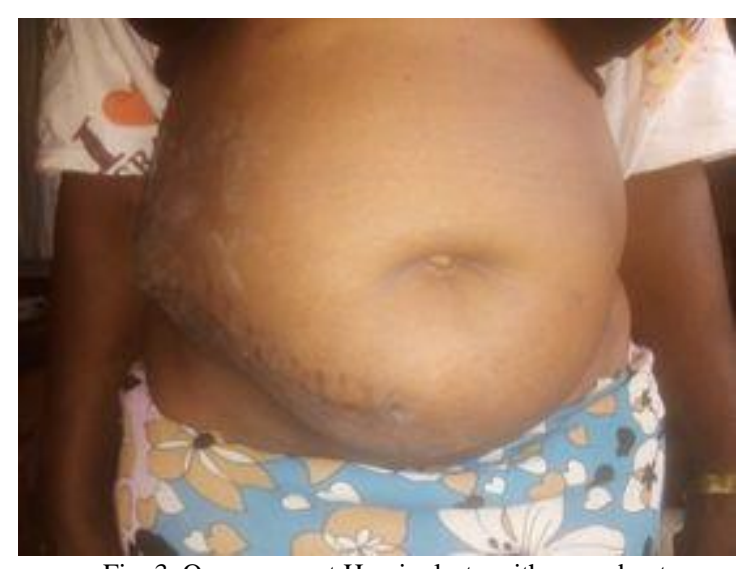

Fig. 3. One year post Hernioplasty with a good outcome.

\section{DISCUSSION}

Incisional hernia after appendectomy, though considered a late complication, may occur within few weeks to months like in our patient [3]-[5]. She had post-operative wound infection which is a significant risk factor. She was not obese, a nonsmoker and had no other known comorbidities [3]-[6].

She had an emergency surgery with an intraoperative diagnosis of perforated appendix in a primary health centre; the qualification of the operating doctor could not be ascertained to access the surgical technique/skill even though the hypertrophic scar appeared to be a Lanz skin incision with an oblique muscle splitting component [3], [5].

Even though oblique incisions are regarded as low-risk incisions, closure of the aponeurosis with interrupted suture instead of continuous suturing, in the presence of afore mentioned risk factors can lead to formation of incisional hernia [1], [3], [7].

Increased intraabdominal pressure like in pregnancy coupled with obliteration of the transversalis fascia in this region thins out the internal oblique muscle leaving the hernia sac directly exposed to the external oblique muscle and fascia which then attenuates laterally. Thus, the ability of the external oblique to hold sutures is compromised and incisional herniation results [2].

Two types of incisional hernias can occur after appendectomy. Commonly, the hernia can pass through all layers of the abdominal wall like in our index case. In the less common interstitial type, the hernia passes through a defect in the transversus abdominis and internal oblique muscles, but not through the intact aponeurosis of the external oblique muscles [4]-[6].

Primary repair of this form of hernia is particularly difficult as a result of the attenuated fascia created by the herniation especially in large defects [2], [4]. They are repaired effectively by standard tension free synthetic mesh through open or laparoscopic approach [4], [7].

In cases where the hernia passes through all layers of the abdominal wall with attendant attenuation and thinning of the abdominal wall laterally like in our index patient, the mesh was placed in an onlay supra-aponeurotic technique anchored to the external oblique fascia [2], [3]. The mesh could also be placed in a subaponeurotic technique either between the peritoneum and the transversus muscle (pre-peritoneal) or between the internal oblique and the external oblique muscle (Sandwich technique) to prevent subsequent lateral herniation [2], [6].

\section{CONCLUSION}

Incisional hernia post open appendectomy though a rare complication, can occur following post-operative infection and is best managed by tension free synthetic mesh repair.

\section{CONFLICTS OF INTEREST /COMPETING INTERESTS}

The authors declare that they have no competing interest.

\section{CONSENT}

A written informed consent for publication of data and pictures was obtained from the patient.

\section{AUTHORS' CONTRIBUTION STATEMENTS}

Both authors prepared the manuscript, conceptualized, and designed the study. All authors read and approved the final manuscript. 


\section{REFERENCES}

[1] Santora TA, Roslyn JJ. Incisional hernia. Surg Clin North Am.1993;73(3): 557-570.

[2] Konstantakos AK, Zollinger RM. Repair of McBurney incisional hernias after open appendectomy. Curr Surg 2000; 57:79-80.

[3] Beltran MA., Cruces KS. Incisional Hernia after McBurney incision. Retrospective case-control study of risk factors and surgical treatment. World J. Surg. 2008;32:596-601.

[4] Maayeh S, Da'meh S, Alamro D, Alhuniti AT, Shboul A, Ammro S. Incisional hernia after appendectomy: A Case Report. Z.U.M.J. 2014;20:421-423.

[5] Hamouda E, Tan B, Peh W. Interstitial incisional hernia following appendectomy. Singapore Med J. 2013;54:531-2.

[6] Edeh AJ, Uchendu UT, Okenwa WO, Ilo AC, Nwangwu CC. Interstitial incisional hernia following open appendectomy: Case report. Indian J Case Reports. 2019;5(3):280-282.

[7] Cassar K, Munro A. Surgical treatment of incisional hernia. $\mathrm{Br}$ J Surg .2002;89:534-545.

[8] Nasrin R, Mohammad C, Morteza B, Ali M, Keyvan K, Amir $\mathrm{S}$ et al. Interstitial incisional hernia following appendectomy: a case report. Int J Med Invest. 2018;7:73-76. 